\title{
Zwischen D'Ancona und Witte: Novati und die Edition der Briefe Dantes
}

Riassunto: Il contributo studia l'apporto di Francesco Novati, ufficialmente incaricato dalla Società dantesca di attendere all'edizione critica delle opere minori latine, alla storia dell'edizione critica delle epistole dopo Witte. L'archivio Novati, conservato a Milano, permette di verificare lo stato del lavoro di edizione, che è risultato ancora molto parziale. L'analisi della documentazione permette di chiarire i criteri e la metodologia di Novati, che non mancò di confrontararsi continuamente, ma non esplicitamente, con le scelte critiche di Witte.

In den Räumen der Biblioteca Braidense in Mailand befindet sich das reichhaltige Francesco Novati-Archiv; es war 1920 in das Archiv der Società Storica Lombarda, deren Präsident Novati gewesen war, gelangt. Drei Jahre zuvor hatte Novatis Bruder Uberto es der Bibliothek geschenkt, die jedoch nicht in der Lage war, es der Öffentlichkeit zugänglich zu machen. ${ }^{1}$ Das 1997 von Elisabetta Colombo erstellte Inventar ist ein unerlässlicher Kompass zur Erschließung dieser beindruckenden Menge von Dokumenten, die unter den persönlichen Archiven von Gegenwartsautoren durch Umfang und Qualität einen besonderen Rang einnimmt. Hinzu kommt, dass in Brera auch Novatis Bücher und sein umfangreicher Briefwechsel aufbewahrt werden, die jedoch fest im Besitz der Bibliothek sind. ${ }^{2}$ Im Jahre 2016 hat eine Ausstellung einen Teil dieser Materialien der Öffentlichkeit bekannt gemacht und so einen Einblick in das intellektuelle und universitäre Leben Mailands um 1900 gegeben, in dem Novati eine herausragende Figur war. ${ }^{3}$

1 Vgl. Elisabetta Colombo, Francesco Novati. Inventario del fondo conservato presso la Società storica lombarda, Milano 1997, S. 42-56.

2 Vgl. ebd.; siehe auch Giulia Chiesa/Donatella Cantele, Francesco Novati: addenda all'inventario del fondo, Milano 2011.

3 Vgl. Leonardo Andreoli/Guido Lucchini/Roberto Tagliani (Hrsg.), Francesco Novati (18591915). Protagonista dimenticato della Milano tra Otto e Novecento. Catalogo della mostra (Milano, Biblioteca Nazionale Braidense, Sala Maria Teresa, 17 marzo - 28 maggio 2016, Milano 2016.

Note: Dieser Beitrag ist aus einem vom ERC (Horizon 2020) geförderten Forschungsprojekt hervorgegangen.

Antonio Montefusco (Ca' Foscari Venezia), E-Mail: astutacolomba@gmail.com 
Diese Ausstellung ist Ausdruck einer neuen Situation, die endlich eine Wiederentdeckung Novatis erlaubt, dessen Ruf lange unter dem Urteil Carlo Dionisottis gelitten hat. Dieser sah in ihm bekanntlich nur einen Gelehrten und gewissermaßen ein Spätprodukt der »scuola storica«; er hatte überdies seine Kompetenz im Gebiet der Romanischen Philologie in Zweifel gezogen, genau jener Disziplin, die Novati lange unterrichtet hatte, erst in Palermo, dann in Genua und schließlich in Mailand. ${ }^{4}$

Studien der letzten Jahre haben auf eine Rekonstruktion dieses kulturellen Milieus gezielt und endlich die Modernität von Novatis Ansatz in der Analyse der mittelalterlichen Literatur aufgewiesen. Trotz seines mangelnden Interesses für die Sprachgeschichte und sprachwissenschaftliche Komparatistik, die ihm Isaia Graziadio Ascoli nie verziehen hat, ${ }^{5}$ hat Novati zu einer geglückten Verbindung von gelehrtem Ansatz und bibliophiler Leidenschaft gefunden. Er hat Büchersammlungen erforscht und unveröffentlichte Texte entdeckt, die er in zwar nicht fehlerfreien, aber soliden Ausgaben zugänglich machte. Auch jemand, der ein scheinbar ganz entgegengesetztes Naturell hat, wie der Romanist Gianfranco Contini, erkannte in ihm einen »Vorläufer moderner Tendenzen« (»precursore di alcuni movimenti di punta«) und zog zum Vergleich das Werk E.R. Curtius' heran, insbesondere im Hinblick auf das Thema der »Kontinuität zwischen der lateinischen und der romanischen Kultur in beiden Richtungen« (»continuità della cultura romanza con la latina nei due sensi«). ${ }^{6}$ Die beiden Hauptwerke Novatis, der von Vallardi publizierte Band Le origini und die monumentale Ausgabe von Coluccio Salutatis Briefwechsel, stellen in der Tat eine umfangreiche Erkundung der Scharnierphase der Überlieferung der klassischen und nachklassischen Kultur in die der Romania dar, die in ihrer ganzen Breite vom zentralen Mittelalter bis zum Humanismus erforscht wird. ${ }^{7}$ Dieser Ansatz war schon in seiner Antrittsvorlesung von 1896 über den Einfluss des lateinischen Denkens

4 Vgl. Carlo Dionisotti, «Scuola storica«, in: Vittore Branca (Hrsg.), Dizionario critico della letteratura italiana, Bd. III, Torino 1974, S. 358.

$5 \mathrm{Zu}$ diesem Thema siehe den unveröffentlichten Brief vom 17. Dezember 1886 von Graziadio Isaia Ascoli an Alessandro D'Ancona, Biblioteca della Scuola Normale di Pisa, Fondo D'Ancona, b. 41. Ich danke Claudio Ciociola, der es mir ermöglicht hat, diese Dokumente einzusehen.

6 Gianfranco Contini, »Un nodo della cultura medievale: la serie `Roman de la Rose $->$ Fiore >Divina Commediar«, in: Vittore Branca (Hrsg.), Concetto, storia, miti e immagini del Medioevo, Firenze 1973, S. 509-542, wieder in ders., Un'idea di Dante. Saggi danteschi, Torino 1976.

7 Francesco Novati, Le Origini, Milano 1900-1909; Epistolario di Coluccio Salutati, Istituto Storico Italiano, Torino 1968-1969, 5 Bde. [I-IV.1-2; 4º, anastatischer Nachdruck der Ausgabe Roma 1891-1911]. 
auf die italienische Literatur enthalten. ${ }^{8}$ Man kann hinzufügen, dass ein besonders moderner Aspekt seiner Tätigkeit die Publikationstätigkeit betraf. Ich beziehe mich auf die Gründung der beiden ältesten heute noch bestehenden italienischen mediävistischen Zeitschriften: 1882 das Giornale storico della letteratura italiana (zusammen mit Renier, Morpurgo und Zenatti) und 1904 die Studi medievali (ebenfalls mit Renier). ${ }^{9}$ Obwohl Novati selbst unter dem Vorwurf litt, ein pedantischer Gelehrter zu sein - »armer kleiner Gelehrter, seit zwanzig Jahren vom Vorwurf der Pedanterie erdrückt, werde ich so sterben wie ich gelebt habe: im Mittelmaß«, schreibt er, entmutigt von den Schwierigkeiten seine Hauptwerke zum Abschluss zu bringen, einmal an D’Ancona ${ }^{10}$-, legen diese beiden Zeitschriften ein beredtes Zeugnis von seiner Herangehensweise ab, die Kultur als allgemeinen Ausdruck einer Zivilisation sieht, in der die Sprachbarriere die geistige Einheit nicht zerstört. In seinem Band Origini schreibt Novati:

Unter den gelehrten Mediävisten herrschte seit langem und herrscht vielleicht auch heute noch [...] die Tendenz, die wir für übertrieben halten, lateinische und volkssprachliche Produktion im Wesentlichen getrennt voneinander zu betrachten, anzunehmen, dass diese nebeneinander gelebt haben, ohne sich fast je gegenseitig zu beeinflussen. Wie zwei Flüsse, die in zwei Betten fliessen und manchmal sogar in demselben (wie bei den beiden Reussflüssen), ohne je ihre Wasser zu mischen. Ich glaube hingegen, dass zwischen den beiden Flussbetten sich mehr Kanäle gebildet haben, und sich mehr Mischungen ergeben haben, als man gemeinhin annehmen will. ${ }^{11}$

Dies ist eine entschieden avantgardistische Position, die auf einer historischen Perspektive beruht, die heute dank einer Reihe von Studien, die sich der Rekon-

8 L'influsso del pensiero latino sopra la civiltà italiana del medio evo, "Annuario della Regia Accademia Scientifico-Letteraria, Facoltà di Filosofia e Lettere in Milano«, wieder: Milano 1897.

9 Vgl. Amedeo Benedetti, »Contributo alla biografia di Francesco Novati«, in: Forum Italicum 46, 2 (2012), S. 253-305 und ders., »Novati, Francesco«, in: Istituto della Enciclopedia Italiana (Hrsg.), Dizionario biografico degli italiani, Bd. 78, Roma 2013.

10 Francesco Novati an Alessandro D’Ancona am 6. Oktober 1898, in: Lida Maria Gonelli (Hrsg.), D’Ancona-Novati, Bd. IV, Pisa 1986-1990, S. 83-85: »Povero eruditucolo, schiacciato da vent'anni sotto l'accusa di pedante, morirò, come sono vissuto, nella mediocrità«.

11 Novati, Le origini (wie Anm. 7), S. 25: »Tra i cultori degli studi medievali invalse tempo addietro e persiste forse ancora oggidì [...] la tendenza, che noi riputiamo eccessiva, a considerare come essenzialmente separata la produzione latina dalla volgare, ad ammettere ch'esse abbiano vissuto accanto l'una all'altra senza compenetrarsi quasi mai: come due fiumi i quali, pur scorrendo in alvei paralleli e talvolta persino in un letto medesimo (come suole avvenire delle due Reuss) non mescolino mai i flutti loro. Io stimo invece che tra i due letti siansi aperti più canali e che la fusione messa tanto in forse abbia avuto effetto con molto maggior frequenza che taluni non si piacciano immaginare«. 
struktion der Literatur und der Geistesgeschichte des Mittelalters als Gegenstand der Sozialgeschichte widmen, wieder ins Blickfeld gerückt ist. ${ }^{12}$ Ich halte es für nicht übertrieben, diesen Ansatz dem der New Cultural History und namentlich Peter Burkes zur Seite zu stellen, der ihn insbesondere auf die neuere Geschichte anwendet. ${ }^{13}$ Was das Mittellatein betrifft, zeigen sich jedoch erhebliche Defizite in Novatis Methode, wie es auch Giovanni Orlandi in seiner Darstellung, dem wohl vollständigsten und leidenschaftlichstem Porträt des Gelehrten, herausgestellt hat. ${ }^{14}$ Orlandi hat vor allem gezeigt, dass Novati einer Idee des »dunklen« Mittelalters verhaftet bleibt; er hat aber auch die Unzulänglichkeiten seiner philologischen Methode aufgezeigt. Novati gehörte einer späteren Generation als Karl Witte an und seine historische Position ist paradoxal: auf der Scheide zwischen wichtigen Neuerungen in den philologischen und historischen Disziplinen, die auch in Italien in jenen Jahren ihre genaueren Konturen empfingen, und dem aufkommenden Idealismus, der diese Art von Forschung in eine Hilfsrolle herabwertete. Die Breite des Arbeitsfeldes der »scuola storica« führte bei Novati, so Orlandi, zu einer übermäßigen Streuung seiner Interessen, die ihn daran hinderte, die aufkommenden Innovationen zu beachten. Das Mittellatein bleibt so eine Berufung, die in einem sehr modernen Sinne zu einer »umfassenden Sicht der Kulturgeschichte und einer sorgfältigen Untersuchung der Texte» (»visione larga della storia culturale e a un'indagine rigorosa dei testi «) führt. ${ }^{15}$

Der Anlass für diesen Beitrag zu Novati liegt darin, dass er (wie Arsenio Frugoni und Francesco Mazzoni) zu den zahlreichen Gelehrten gehört, die eine Edition der Briefe Dantes vorbereitet haben, ohne sie zu publizieren. ${ }^{16}$ Im vorliegenden Text findet auf der Grundlage der Materialien des Fondo Novati in der Biblioteca Braidense eine erste Erkundung zu dieser mehrmals angekündigten aber nie erschienenen Ausgabe von Dantes Briefen statt. Zuvor sollten wir uns jedoch der Frage nach der Beziehung zwischen Novati und Witte zuwenden, dem unbestrittenen Patron der Erforschung von Dantes Briefen, die er auch, wie Enrica Zanin in mehreren Beiträgen gezeigt hat, in indirekter Weise beeinflusst hat. ${ }^{17}$ Ein

12 Vgl. zum Beispiel den Band von Ronald G. Witt, The Two Latin Cultures and the Foundation of Renaissance Humanism in Medieval Italy, Cambridge 2012.

13 Für einen Überblick siehe Peter Burke, La storia culturale, Bologna 2006.

14 Vgl. Giovanni Orlandi, "Francesco Novati e il Medioevo latino. Storia di una vocazione«, in: Gennaro Barbarisi/Enrico Decleva/Silvia Morgana (Hrsg.), Milano e l'Accademia scientifico-letteraria. Studi in onore di Maurizio Vitale, Bd. I, Milano 2001, S. 465-600.

15 Ebd., S. 596.

16 Vgl. Antonio Montefusco, »Le `Epistole di Dante: un approccio al corpus«, in: Critica al testo 14, 1 (2011), S. 401-457, S. 409-416.

17 Siehe ihren Beitrag in diesem Band des Dante-Jahrbuchs. 
direkter Kontakt zwischen den beiden Gelehrten, die zwei verschiedenen Generationen angehörten, ist nicht belegt. Es ist jedoch bemerkenswert, dass in der schwierigen Gründungsphase des Giornale storico della letteratura italiana der junge Mitbegründer seinen Lehrer Alessandro D’Ancona mehrmals aufforderte, für die Zeitschrift zu schreiben und ihm bekannte italienische und ausländische Gelehrte zur Mitarbeit einzuladen; in diesem Zusammenhang erwähnte er auch Witte. ${ }^{18}$ D'Ancona antwortete hierauf nicht, gab aber nach Wittes Tod einen Artikel über das Werk des deutschen Gelehrten für die Zeitschrift in Auftrag. Dieser bizarre Beitrag wurde von Carlo Vassallo (1828-1892), Domherren der Kathedrale von Asti und Gymnasiallehrer, der mit den hervorragendsten Danteforschern seiner Zeit in Kontakt stand, verfasst. Er fand nicht das Gefallen der Direktoren der Zeitschrift, da er zu emphatisch war, und wurde in La rassegna nazionale veröffentlicht. ${ }^{19}$ Novati bat D'Ancona um Auskünfte über den Autor ${ }^{20}$ und D'Ancona gab zu, dass »der Beitrag Vassallos von mir in Auftrag gegeben wurde, da ich wünschte, dass jemand ausführlich Wittes Dantestudien darstellt. Was er geschrieben hat, weiss ich nicht; aber ich glaube, dass er, trotz einiger

18 »Ich muss Sie um einen anderen Gefallen bitten: einen, den sie mir versprochen haben, d.h. an verschiedene ausländische Literaturforscher, die Sie kennen, zu schreiben, damit diese meine Einladung zur Mitarbeit, wie sie es hoffentlich verdient, annehmen. Ich beziehe mich auf Paris, Wesselofski und noch andere, um nicht alle bekannten Namen aufzuzählen. Glauben Sie, dass man sich an sie wenden kann? Und ich wäre Ihnen sehr verbunden, wenn Sie mich auf mir nicht bekannte Namen hinweisen. So könnten Sie an d'Ovidio, Canello, Villari schreiben. Witte? Kennen sie Ihn? Und Voigt und Böhmer. Ich will nicht indiskret sein, bin mir aber sicher, auf Ihre Hilfe zählen zu können«; Brief Novatis an D’Ancona vom 14. Oktober 1882, in: Gonelli (Hrsg.), D’Ancona-Novati (wie Anm. 10), Bd. I (Carteggio d'Ancona; 7), S. 230. Über die erste Phase der Geschichte der Zeitschrift, siehe Benedetti, »Contributo« (wie Anm. 9), S. 256-260: »E di un altro favore io debbo farLe dimanda: di un altro che Ella già mi promise, di scrivere cioè a varî fra $i$ letterati stranieri che Ella conosce perché mi vogliano accogliere come speriamo debba meritare l'invito a collaborare. Intendo del Paris, del Wesselofski e di quant'altri per non star qui a tenere una serie di nomi noti Ella crede possano essere interpellati. Anzi mi farà un piacere se alcuni che io non conoscessi me ne mettesse sott'occhio. Così fra noi Ella dovrebbe scrivere al d'Ovidio, al Canello, al Villari. Il Witte Ella lo conosce? E il Voigt e il Böhmer? Non vorrei essere indiscreto; ma se Ella può giovarci son sicuro lo farà «.

19 Carlo Vassallo, »Sulla vita e sugli scritti di Carlo Witte«, in: La Rassegna Nazionale XVI (1884), S. 601-635; XVII (1884), S. 167-207.

20 »Welchen Wert hat die Schrift von Vassallo über Witte, der er dem Giornale anbietet? Sie scheint mir sehr lang zu sein? Ist sie gut?«; Brief Francesco Novatis an D’Ancona vom 1. November 1883, in: Pio Rajna/Francesco Novati, Carteggio (1878-1915). Tra filologia romanza e mediolatina, hrsg. von Guido Lucchini, Milano 1995, S. 344: „Che valore ha un lavoro del Vassallo sul Witte che propone al Giornale? Par molto lungo. Ė buono?«. 
Übertreibungen in der Manier Giulianos ein guter Mann ist «. ${ }^{21}$ Der Vorgang ist bezeichnend: D’Ancona war sich der Rolle Wittes für die Dante-Studien bewusst, und es gab einen Kontakt zwischen den beiden Gelehrten. In der Scuola Normale Superiore in Pisa sind Teile eines Briefwechsels über Dante betreffende Themen erhalten, die eine Veröffentlichung verdient hätten. ${ }^{22}$ Ebenso bezeichnend ist jedoch, dass die jungen und ehrgeizigen Gründer des Giornale (vor allem Renier und Novati) bereit waren, auf den Nachruf zu verzichten, um der Ausrichtung der neuen Zeitschrift nicht zu schaden. Um etwas mehr darüber zu erfahren, was für eine Vorstellung Novati sich über Wittes Forschungen zu Dantes Briefen gemacht haben könnte, müssen wir uns im Fondo des Archivs umsehen.

Der Auftrag an Novati, Dantes Briefe zu edieren, kam von seinem Lehrer D'Ancona und kann wohl damit erklärt werden, dass Novati sich mit Coluccio Salutati befasste und seine Monographie über die Jugend des Florentiner Kanzlers, aber vor allem einen Teil seines imposanten Briefwechsels veröffentlicht hatte. ${ }^{23}$ Da D’Ancona ihn schon 1894 erwähnt, erging der Auftrag wahrscheinlich kurz nach der Veröffentlichung des ersten Bandes von Salutatis Briefwechsel. Wenige Jahre später fragt D’Ancona Novati nach den Fortschritten seiner Arbeit, dieser aber teilt mit, er komme nur langsam voran. ${ }^{24}$ Pio Rajna, ein weiterer

21 Ebd., S. 346: »il lavoro del Vassallo fu fatto a istanza mia, desiderando che qualcuno parlasse largamente degli studj Danteschi del Witte. Che cosa egli abbia fatto non so: ma spero bene, ché, salvo qualche fronda giulianesca, il V. è un brav'uomo«.

22 Es handelt sich um sieben Briefe Wittes an D’Ancona, die in der Bibliothek der Scuola Normale Superiore in Pisa aufbewahrt werden: Fondo D'Ancona, s. II [Briefe der Korrespondenzpartner], ins. 45, b. 1429, (Dezember 1854-März 1876; zwei dieser Briefe sind ediert in Alessandro D’Ancona, Pagine sparse di letteratura e di storia. Con appendice »dal mio carteggio«, Firenze 1914. Vgl. zum Briefwechsel zwischen Witte und D’Ancona den Beitrag von Angelo Colombo in diesem Band.

23 Vgl. Francesco Novati, La giovinezza di Coluccio Salutati (1331-1353). Saggio di un libro sopra la vita, le opere, i tempi di Coluccio Salutati, Torino 1888; Epistolario (wie Anm. 7).

24 »Ich habe einen Brief von Torrigiani erhalten, in dem ich gefragt werde, wie es um meine Forschungen für die Veröffentlichung des Briefes (sic) und der Eklogen Dantes steht. Ehrlich gesagt, sind meine Forschungen so wenig weit gediehen, dass ich nicht so recht weiss, was ich antworten soll. Wenn dich jemand danach fragt, kannst Du sagen - falls Dir das nichts ausmacht dass ich dabei bin, die Materialien für die Edition zu sammeln, dass es aber nur langsam vorangeht «; Francesco Novati an Pio Rajna, am 9. Juni 1897, in: Rajna/Novati, Carteggio (wie Anm. 20), S. 194: »Io personalmente ho poi ricevuto una lettera del Torrigiani in cui mi si chieggono notizie della condizione nella quale son le indagini da me avviate per la pubblicazione della Epistola (sic) e delle Egloghe Dantesche. A dir vero, le mie ricerche sono finora così poco avanzate, ch'io non so troppo che rispondere. E se per caso tu udisse domandarne, puoi - ove non ti spiaccia - accennare che mi sto bensì occupando di raccogliere i materiali per l'edizione; ma che la cosa procede lentamente assai«. 
Schüler D’Anconas und - wenn auch etwas älterer - Weggefährte Novatis verfolgte die Fortschritte seiner Arbeit. Diese kam jedoch aus verschiedensten Gründen nur sehr mühsam voran. Der Hauptgrund mag wohl in Novatis Arbeit an Coluccio Salutati liegen; hinzu kommt die erdrückende Aufhäufung von Nebenpisten in den Forschungen eines Gelehrten, dessen Arbeitsfeld sich von der griechischen Literatur bis zu den zeitgenössischen Literaturen erstreckte.

Dieselben Schwierigkeiten kennzeichnen auch die in diesem Zusammenhang entstehenden Veröffentlichungen. Novati publizierte nur zwei Artikel über Dantes Briefe und diese kurz hintereinander. Der erste ist ein allgemeiner Überblicksartikel, der im Rahmen einer Vortragsreihe zu Dantes »opere minori« in Orsanmichele 1905 entsteht. ${ }^{25}$ Der andere Artikel erscheint wenig später, im Jahr 1908, in einem Band über Dante und die Lunigiana und behandelt die Epistel IV an Moroello Malaspina. ${ }^{26}$ Das Publikationsdatum ist aber irreführend, da dieser Band schon seit langem in Vorbereitung war und Novati mehrmals erwähnt, vom Verleger in dieser Angelegenheit gemahnt worden $\mathrm{zu}$ sein. ${ }^{27}$ Hierzu liefern die Archivdokumente wesentliche Hinweise: sie erlauben es zum einen zu verstehen, wie weit die Arbeit für die Edition gediehen war, zum anderen enthalten sie wertvolle Elemente zur Kenntnis von Novatis Methode. Um zu beiden Fragen abschließende Antworten zu erhalten, müsste man die Forschung vertiefen und insbesondere die große philologische Unternehmung der Herausgabe von Coluccios Briefwechsel mit einbeziehen. Ich kann hier nur einige Hinweise geben und behalte mir Vertiefungen für zukünftige Publikationen vor.

Die nie abgeschlossene und Jahrzehnte währende Arbeit Novatis an Dantes Briefen ist im Faszikel 146 des Umschlages 31 dokumentiert, der mit »Epistole di Dante « betitelt ist. ${ }^{28}$ Er umfasst sechzehn nummerierte Ordner, deren Inhalt in zwei große Sektionen aufgeteilt werden kann. Die erste umfasst Notizen und Materialien zur Kontextualisierung von Dantes Briefwechsel (Ordner 1 bis 2 sowie 15 und 16). Es handelt sich hierbei um Blätter verschiedener Größe, die vermutlich zuerst nach und nach beschrieben wurden und dann von Novati zusammen geordnet wurden in einem Heft, auf dem von Novati selbst der Titel »Appunti

25 Francesco Novati, Le epistole. Conferenza letta nella sala di Dante in Orsanmichele, Firenze 1905 (wieder in Novati, Freschi e minii del Dugento, Milano 1908, S. 329-361).

26 Wir verwenden die Ordnung und Zählung der Briefe Dantes, die sich seit der in der Edizione Nazionale von 1921 von E. Pistelli publizierten Ausgabe durchgesetzt hat.

27 Francesco Novati, „L'Epistola di Dante a Moroello Malaspina«, in: Ulrico Hoepli (Hrsg.), Dante e la Lunigiana. Nel sesto Centenario della venuta del Poeta in Valdimagra, MCCCVI-MCCCCVI, Milano 1908, S. 505-542; siehe die Avvertenza, wo vermerkt ist, dass sich bei der Vorbereitung des Buches »einige Verzögerungen« ergeben hatten (S. IX: »dovette subire alcune soste«).

28 Siehe die Beschreibung bei Colombo, Francesco Novati (wie Anm. 1), S. 110-112. 
generali sulle Epistole« vermerkt ist. Es gibt einige Ordnungselemente (so zum Beispiel in 31.146.1 eine Liste der Briefe mit dem jeweiligen Incipit und der Liste der Handschriften, natürlich nur der damals bekannten: $" 3$ cod. e Marciana «, ist am oberen Rand des Rückeinbandes dieses Verzeichnisses vermerkt). ${ }^{29}$ Trotzdem erscheint die Sammlung insgesamt chaotisch und macht keine Unterschiede zwischen den bibliographischen Notizen, die auf die Titel beschränkt werden; auf dem ersten Blättchen findet sich nur der Titel der von Witte herausgegeben Ausgabe der Briefe von 1827. ${ }^{30}$ Auch wenn es vielleicht heißt, diesem Detail zu viel Bedeutung beizumessen, scheint mir dieser Eintrag klar zu belegen, dass auch für Novati der Zugang zu Dantes Briefen die Arbeiten Wittes waren, die in der modernen Danteforschung lange unterbewertet oder kaum beachtet wurden. Die Mehrzahl der Blätter enthält umfangreiche Exzerpte aus der wissenschaftlichen Fachliteratur zur Erforschung des Kontextes von Dantes Briefen; hierzu gehört die Lektüre, unvermeidlicherweise unvollständig und am direkten Nutzen orientiert, des Codice diplomatico dantesco von Passerini-Biagi (aus dem Novati Informationen zu den Jahren 1303-1304, also im Zusammenhang mit den ersten beiden Briefen Dantes, exzerpiert) und des magnum opus von Zingarelli (ebenfalls hauptsächlich für denselben Zeitraum konsultiert). ${ }^{31}$ Im zweiten Ordner finden sich Exzerpte zur Geschichte des dictamen und der Schulen in der Toskana. Hier finden sich auch Listen von magistri in kleineren Zentren, insbesondere Siena und Arezzo. Ebenfalls gewichtig sind die Aufzeichnungen zum cursus und zum Latein Dantes (mit einem Schwerpunkt auf den Briefen, aber auch mit Anmerkungen zum De vulgari, das Novati wahrscheinlich als den Briefen näherstehend empfand als die restlichen Schriften Dantes auf Latein). Hinzu kommt die Nieder-

29 Die Handschriften, die Dantes Briefe überliefern sind folgende: Boccaccios Zibaldone Laurenziano (Firenze, Biblioteca Medicea Laurenziana, Pl. XIX.8 = L), der die Briefe III, XI, XII enthält; Biblioteca Apostolica Vaticana, Pal. Lat. 1729 = V, Briefe I, II, IV, V, VI, VII, VIII, IX, X; Roma, Biblioteca Nazionale Centrale Vittorio Emanuele, S. Pantaleo 8 (101) = P Briefe V und VII (letzterer auch in der Volkssprache); Venezia, Biblioteca Nazionale Marciana, Latino XIV 115 (4710) = M, nur Brief VII. Novati konnte noch nicht die Handschrift Siena, Biblioteca Comunale degli Intronati, F V 9 = S, berücksichtigen, die einen Teil des Briefes VII überliefert, da diese erst seit 1939 bekannt ist.

30 Karl Witte (Hrsg.), Dantis Alligherii Epistolae quae exstant cum notis Caroli Witte..., Pavia 1827.

31 Der »Codice« Passerini-Biagi wurde bekanntlich in Faszikeln zwischen 1895 und 1911 veröffentlicht. Novati schaffte die ganze Serie an: Guido Biagi/Guiseppe L. Passerini, Codice diplomatico dantesco. I Documenti della vita e della famiglia di Dante Alighieri, riprodotti in fac-simile, trascritti e illustrati con note critiche, monumenti d'arte e figure, Roma/Firenze 1895-1911: disp. I, mag. 1895; II, ag. 1897; III, giu. 1898; IV, dic. 1898; V, giu. 1900; VI, dic. 1900; VII, mar. 1903; VIII, giu. 1903; IX, set. 1904; X, dic. 1904; XI, mar. 1905; XII, mag. 1909; XIII, feb. 1910; XIV, gen. 1911. Siehe auch Nicola Zingarelli, La vita, i tempi e le opere di Dante, Milano 1931 (erste Ausgabe: 18981902). 
schrift des schon erwähnten Vortrages über Dantes Briefe, den Novati 1905 im Dantesaal von Orsanmichele hielt.

Die von Novati gesammelten Materialien fließen in diese lectura ein, die von den Danteforschern wenig beachtet und zitiert wird, die aber ein vielversprechendes Arbeitsprogramm skizziert. Novatis Ausführungen, wenn auch von einer negativen Bewertung der "mittelalterlichen« Natur des Lateins des dictamen bestimmt - eine negative Sicht des Mittelalters, die paradoxerweise bei Novati häufig anzutreffen ist und die vielleicht auf seine altphilologische Ausbildung und seinen Lehrer Piccolomini zurückzuführen ist ${ }^{32}$-, betreffen vor allem den Kontext und die Kulturgeschichte: Dantes Ausbildung, mit einer bemerkenswerten Schilderung des Rhetorikunterrichtes in der Toskana, sowohl in Florenz als auch in kleineren Städten, und der Rolle Brunetto Latinis; eine knappe Vertiefung zu den stilistischen Eigenschaften von Dantes Briefen, die auch im Lichte des De vulgari betrachtet werden; und schließlich die Widerlegung der Argumente, die gegen die Authentizität der Briefe vorgebracht worden waren. In einer anderen Veröffentlichung habe ich die Bedeutung dieses Arbeitsprogramms hervorgehoben, das, wenn man es im Lichte der wichtigsten Resultate der jüngeren Forschung zur ars dictaminis betrachtet, als besonders relevant erscheint. ${ }^{33}$ Ich kann hier diese Einschätzung nur bekräftigen und zugleich unterstreichen, dass dieser Vortrag im Lichte der Materialien zu ihrer Vorbereitung einen klaren Eindruck von Novatis Methode und seinem Editionsvorhaben vermittelt.

Was den ersten Aspekt angeht, wird deutlich, wie die Textanalyse die Gelegenheit für eine reichhaltige Serie von Vertiefungen zu kulturellen und historischen Fragen darstellt. Der Text, oder genauer: der literarische Text, wird von einer großen Zahl von Detailbeobachtungen geradezu überdeckt; diese fügen sich nicht kohärent zusammen, sondern stellen eher ein Instrument dar, um die Epoche zu verstehen, in der der Text entstanden ist. Diese »gelehrte« Herangehensweise wird, wie ich schon sagte, heute immer noch als zu weit ausholend und geradezu chaotisch angesehen; ich glaube hingegen, dass sie die Grundlage für eine historisch treffendere Textinterpretation legt. Was das Stadium angeht, an dem sich Novatis Editionsvorhaben befand, kann man gewiss feststellen, dass im Jahre 1905 auch diese Arbeit zum Kontext weit von einer Vollendung entfernt war. Novati spricht übrigens in seinem an Rajna gerichteten Brief aus dem Jahre

32 Siehe die von Enea Piccolomini, »Sulla essenza e sul metodo della filologia classica (continuazione e fine)«, in: Rivista europea 6, 4 (1875), S. 104-106 formulierten Überlegungen.

33 Vgl. Antonio Montefusco, »Le lettere di Dante: circuiti comunicativi, prospettive editoriali, problemi storici«, in: Giuliano Milani/Antonio Montefusco (Hrsg.), Le lettere di Dante. Ambienti culturali, contesti storici e circolazione dei saperi, Berlin 2020, S. 1-39. 
1902 nur von den »schrecklichen Schwierigkeiten« (»terribili difficoltà») bei der Erstellung des kritischen Textes ${ }^{34}$ und erwähnt den Kommentar nicht.

Überprüfen wir diese Aussage anhand der Dokumente. Die zweite Makrosektion des Faszikels 146 enthält die Ordner 3 bis 14. In 11 modernen Ordnern werden die originalen Dokumente aufbewahrt, von etwas kleinerem Format und verschiedenem Erhaltungsgrad. Die 11 Ordner enthalten Material zu jedem der damals bekannten Briefe von Dante und sind mit römischen Ziffern nummeriert. Die meisten betreffen essenzielle Informationen: das Datum des Briefes, sein incipit, die Handschrift(en), die sie überliefert/n mit der zughörigen Ortsangabe. Hinzu kommen manchmal Notizen verschiedener Art; so wird für den Brief an Niccolò da Prato (Brief I nach der heutigen Nummerierung) vermerkt, dass der Anführer der Florentiner »Bianchi« von Bruni als Alessandro da Romena identifiziert wird, von Barbagallo als Aghinolfo. Für den Brief anlässlich des Todes Alessandros findet sich eine Skizze zum Stammbaum der conti Guidi, usw. Im Ordner befindet sich ein gefaltetes A3-Blatt. Auf der einen Seite ist von Hand mit blauer Tinte am oberen Rand in Großbuchstaben und größerer Schrift vermerkt »Epistolae Dantis Alagerii«; am unteren Rand, in kleinerer Schrift: »Skript« mit fortschreitender römischer Nummerierung der Briefe. Auf der anderen Seite findet sich in einer eleganten Kursivschrift mit blauer Tinte die Transkription des Textes des jeweiligen Briefes. Am oberen Rand wird »Epistolae Dantis Alagerii« wiederholt, während am unteren Rand »Epistola Ia« usw. steht. An der Ecke jeder Vorder- und Rückseite werden die Blätter von I bis XIV fortlaufend nummeriert.

Ich muss auf eine eingehende Analyse verzichten und komme sogleich zum konkreten Inhalt dieser Makrosektion, um zu verstehen, bis zu welchem Punkt die Edition gediehen war, und um die Methode Novatis zu charakterisieren. Was den ersten Aspekt angeht, müssen wir überprüfen, ob das, was er Rajna 1902 schreibt - in dem Brief, in dem er sagt, dass er sich die Reproduktionen der Handschriften besorgt und den kritischen Text vorbereitet hat - , mit den uns

34 »Ich bin in Verlegenheit, dir zu antworten, da meine Arbeit seit einiger Zeit festgefahren ist. Hier jedenfalls die Lage: ich habe eine photographische Reproduktion der drei Handschriften anfertigen lassen. Diese enthalten ganz oder teilweise (der Vaticano Palatino, der Vitt. Emanuele, der Laurenziano) Dantes Briefe, und ich bereite den kritischen Text langsam vor, inmitten grösster Schwierigkeiten, die der ausserordentlichen Unzuverlässigkeit der Handschriften geschuldet sind. Die Arbeit an den Egloghe ist weiter vorangeschritten" (Rajna/Novati, Carteggio [wie Anm. 20], S. 215: »In quanto a ciò che chiedi io sono alquanto imbarazzato a darti una risposta, perché da qualche tempo i lavori miei si sono arenati. Ad ogni modo, eccoti la condizione mia. Ho fatto riprodurre fotograficamente i 3 codd. Che dànno le lettere di Dante tutte o in parte [il Vaticano Palatino, il Vitt. Emanuele, il Laurenziano] e sono venuto preparando pian piano, tra terribili difficoltà prodotte dalla somma corruzione dei codd., il testo critico. Il lavoro è più avanzato per le Ecloghe«). 
bekannten Dokumenten vereinbar ist. Unser Ausgangspunkt ist, dass diese Aufzeichnungen unvollständige Kollationen enthalten. Die mise en page des Textes, der in blauer Tinte transkribiert, in Abschnitte unterteilt und mit Zeichensetzung

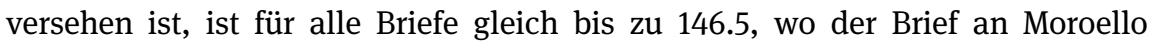
Malaspina transkribiert ist und die ganze Seite ausfüllt. Für die anderen Briefe diente der Rand dafür die Varianten in kleinerer Schrift und schwarzer Tinte zu verzeichnen. Die Arbeit der Kollation war jedoch nicht abgeschlossen. Zunächst stellt man fest, dass für drei Briefe (146.4: Brief II, 146.8: Brief VI, und 146.11: Brief XII) die Ränder leer sind. In den anderen Briefen ist die Situation unterschiedlich und reicht von den Briefen IV (146.5) und XI (146.10), für die die Kollation reichhaltig ist und fast abgeschlossen zu sein scheint, bis zu sehr beschränkten Kollationen (u.a. überraschenderweise für den Brief Ia; 146.3). Bei den Briefen, die in mehreren Handschriften überliefert sind (V und VII), stammen die am Rande verzeichneten Varianten nur aus der Handschrift Roma, S. Pantaleo 8. Das führt uns natürlich zu der Frage, welche Textgestalt Novati für seine Transkription gewählt hat. Dies ist eine zentrale Frage, die auch für die Methode des Mailänder Gelehrten erhellend ist.

Der Basistext ist nicht, wie man annehmen könnte, eine interpretative Transkription des wichtigsten Manuskriptes für Dantes Briefe, $V$. Um das festzustellen, genügt es, die Varianten des Briefes I durchzusehen: hier hat Novati im Text bei I,1 »Nicholao« und vermerkt am Rande mit schwarzer Tinte »Richo«, was die fehlerhafte Variante des Vatikanmanuskriptes ist (die Varianten sind nur für die salutatio des Briefes vermerkt). Ein ähnliches Bild ergibt sich im Hinblick auf den Brief XI (146.10): hier sind am Rande die Varianten (dieses Mal fast alle) der Handschrift $L$ aufgelistet. Der Basistext muss deswegen wohl aus einer gedruckten Ausgabe stammen. Da Novati hierzu keine Angaben macht, habe ich einen Vergleich mit modernen Editionen angestellt, insbesondere mit denen, die sich in dem von P. Fraticelli herausgegebenen und kommentierten Band I Il Convito di D. Alighieri e le epistole finden; die Textgestalt der Briefe in dieser Ausgabe ist, wie der Herausgeber informiert, von Karl Witte. Es handelt sich um eine Edition von herausragender Bedeutung, die entscheidend zur Fixierung des VulgataTextes beigetragen hat. ${ }^{35}$ Dass dies jedoch nicht der von Novati zugrunde gelegte Text ist, bemerkt man schon an der Anordnung der Texte. Diese stimmt mit derjenigen der Ausgabe Fraticelli-Witte nur bis zu 146.10 überein, wo der Brief an die Kardinäle (jetzt XI) als »Dispensa VIII« bezeichnet wird; der Brief VIII bei

35 Vgl. Pietro Fraticelli (Hrsg.), Il Convito di D. Alighieri e le epistole, Bd. I, Firenze 1857; über die Mitarbeit Wittes an dieser Ausgabe siehe den Beitrag von Enrica Zanin in diesem Band. 
Fraticelli-Witte ist der Dante zugeschriebene, aber nicht von ihm stammende Brief in der Volkssprache an Guido da Polenta (bei Novati »Dispensa XIV«).

Das Geheimnis wird durch die Bezüge auf die Gedichte, die den Briefen an Moroello und Cino beigefügt sind, gelüftet; Novati bezieht sich auf diese als Canzone IX (146.5) beziehungsweise als Sonett XXXVI (146.6) in der Zählung der Ausgabe aller Werke Dantes von E. Moore aus dem Jahre 1894. Und in der Tat, sowohl die Anordnung der Briefe als auch die Textgestaltung bis hin zur Interpunktion sind mit der Oxford-Ausgabe identisch. ${ }^{36}$ Man bemerke zudem, dass in Heft 146.13 Novati für die Briefe an Margarete von Brabant, die Dante im Namen der Gherardesca der Conte Guidi verfasst, die Textgestalt der Ausgabe von Torri von 1842 wählt, wie er in einer langen Fußnote selber darlegt. ${ }^{37}$ Dies erklärt sich aus dem Umstand, dass Moore und Fraticelli beide den Text dieser kurzen Briefe nicht abdrucken, da erhebliche Zweifel an ihrer Authentizität herrschten. Es ist mithin interessant, dass Novati Dante auch diese im Dienste einer Kanzlei verfassten Briefe zuschreibt, wie er es auch in der Lectura von 1905 tut. Dies gehört zu den wichtigen Errungenschaften der Beschäftigung Novatis mit diesen Texten. ${ }^{38}$

Da es hier unmöglich ist, eine umfassende Analyse der Kollation Wittes vorzustellen, beschränke ich mich auf Beispiele aus zwei Heften. 146.5, das die Aufzeichnungen und das Skript zum Brief an Moroello enthält, ist das umfangreichste Heft, da es der Vorbereitung des 1908 veröffentlichten Artikels diente. Aber auch nach dieser Publikation fügte Novati Material hinzu, da hier auch die unveröffentlichten Briefe von Busnelli und Pio Rajna aufbewahrt sind, wo diese $\mathrm{zu}$ dieser Veröffentlichung Stellung nehmen. Wie schon zuvor erwähnt, ist die mise en page hier eine andere. Novati hat mehrere Passagen unterstrichen und interlinear alternative Lesarten vermerkt. Es handelt sich jedoch nicht um die Kollation des von Moore editieren Textes mit der Handschrift, sondern um ein schon weiter fortgeschrittenes Arbeitsstadium, da die interlinear notierten Lesarten diejenigen sind, die Novati in seinem Artikel verteidigt. Ich wähle zwei Beispiele. Im Paragraph IV.i.2 liest die Handschrift »quam affectus gratuitatis dominatis« (es geht um die Beziehung zwischen dem Autor des Briefes und dem Adressaten). Witte hatte die schwierige Syntax mit einem eher schwerwiegenden Eingriff emendiert: "gratitutudinis«, den Moore übernimmt. Novati hingegen ver-

36 Vgl. Dante Alighieri, »Epistole«, in: Edward Moore (Hrsg.), Tutte le opere di Dante Alighieri, Oxford 1894.

37 Vgl. Alessandro Torri (Hrsg.), Epistole di Dante Allighieri edite e inedite. Aggiuntavi la dissertazione intorno all'acqua e alla terra e le traduzioni rispettive a riscontro del testo latino con illustrazioni e note di diversi, Livorno 1842.

38 Vgl. Novati, Le epistole (wie Anm. 25), S. 18. 
teidigt die Lesart der Handschrift auf der Grundlage des indirekten Zeugnisses der Mavortis Miles Boccaccios, wo dieser schreibt: »Post diutinam lassitudinem gratiam merui dominantis«. Leider belässt es Novati dabei und behält Wittes Korrektur »dominantis « für »dominantur «. ${ }^{39}$ In der benivolentiae captatio übernimmt Novati die Korrektur von »oraculi seriem« nicht. In seinem Aufsatz wird er erneut die Gelegenheit ergreifen, eine lange Liste von Korrekturen zurückzuweisen (u.a. Wittes »oratiunculae«). Um die Lesart der Handschrift zu verteidigen, wird erneut Boccaccio und dessen Brief II angeführt. ${ }^{40}$

Insgesamt zeichnet sich Novatis Herangehen dadurch aus, dass er versucht, mit den exzessiven Eingriffen der vulgata (wie sie auch Witte praktiziert) Schluss zu machen, um mit Aufmerksamkeit die Lesarten, so wie sie die Handschriften überliefern, zu würdigen. Sein wichtigstes Instrument ist hierbei die lexikographische Analyse, für die er sich auf seine ausserordentliche Kenntnis der mittellateinischen Tradition stützen konnte. Hinzu treten zahlreiche Beobachtungen, die aus der Sprache Dantes kommen bzw. aus der Analyse des Kontextes, in dem sich die Personen bewegen, die in den Briefen vorkommen. Hier ein Beispiel aus den Papieren des Archives, das noch nie veröffentlicht wurde. In 146.3 betreffen Novatis Beobachtungen und Anmerkungen nicht nur die problematische und heute noch umstrittene Variante »rubeant « (I, ii. 5; Dante spricht von den Waffen der Weißen, die rötlich erscheinen), die er in seiner Lectura von 1905 bespricht. ${ }^{41}$ Nicht minder interessant sind seine Beobachtungen über das Verb des Satzes »sub paterna monitione pollicetur« (I.ii.4). Das Vatikan-Manuskript liest falsch »polux«; die Verbesserung ist eine Korrektur von Torri, die Witte-Fraticelli übernehmen. In seinen Aufzeichnungen gelangt Novati anhand einer genauen lexikalischen und grammatikalischen Analyse (die u.a. auch Papias herbeizieht) zu der Vermutung, dass die Verbform vom aktiven "polluceo« (die Dante auch in De vulgari eloquentia II.i.1 in der Form »polluximus« benutzt) mit der Bedeutung »versprechen« stammen muss. Davon kann man ein »polluxit« ableiten, das Novati nicht ausdrücklich erwähnt, das aber heute die allgemein akzeptierte Lektion ist. ${ }^{42}$

39 Ders., "L'epistola di Dante« (wie Anm. 27), S. 520-526, und vor allem die treffende Fußnote auf S. 540.

40 Vgl. ebd., S. 527-529 und 541. Der corpus von Boccaccios Briefen ist ediert in Giovanni Boccaccio, »Epistole«, hrsg. von Ginetta Auzzas, in: ders., Tutte le opere, Bd. V/1, hrsg. von Vittore Branca, Milano 1992, S. 493-846.

41 Siehe Novati, Le epistole (wie Anm. 25), S. 33, Nr. 60.

42 Siehe Dante Alighieri, »Epistole«, in: Nuova Edizione Commentata delle Opere di Dante, Bd. V, hrsg. von Marco Baglio/Luca Azzetta/Marco Petoletti/Michele Rinaldi, Roma 2016, S. 65, n. ad locum. 
Wir müssen noch klären, wofür das in der 31.146 gesammelte Material bestimmt war. Man kann annehmen, dass es nicht nur der Vorbereitung der kritischen Ausgabe diente, sondern auch als Unterrichtsmaterial dienen sollte, von dem die Studenten Kopien anfertigen konnten. Dafür spricht, dass zweimal der Begriff »Skript « benutzt wird. Beide Male liegt keine Kollation vor (es handelt sich um den Brief an Kaiser Heinrich den VII., jetzt Brief VII, in 146.9 und denjenigen an die Kardinäle, jetzt XI, in 146.10). Es ist sehr wahrscheinlich, dass Novati dieses »Skript « in seinen Universitätsvorlesungen in »Letterature neolatine« im Jahr 1898-99 benutzte, als er $\mathrm{zu}$ Le epistole di Dante: esercitazioni critiche $e$ filologiche sopra il testo di esse las und dann im Jahre 1904-05 zum Thema Studi sulla ricostituzione critica del testo delle Epistole dantesche. ${ }^{43}$

Abschließend kann man sagen, dass das im Archiv vorhandene Material das bestätigt, was Orlandi über die Defizite von Novatis Methode gesagt hat. Die philologische Arbeit an Dantes Briefen, wenn auch in einem Anfangsstadium befindlich, gleicht methodisch derjenigen an den Briefen Coluccio Salutatis: der Text wird mit den hoch entwickelten Instrumenten der sprachwissenschaftlichen und historischen Gelehrsamkeit seziert. Von der textkritischen Seite her ist Novatis Vorgehen hybrid und nicht strikt an Lachmanns Methode orientiert. Wir sind gewiss noch von der »nuova filologia« entfernt. Der Versuch, zu den Lesarten der Handschriften zurückzukehren und die Korrekturen, die sich angesammelt hatten, einer radikalen kritischen Prüfung zu unterziehen, darf dennoch nicht unterbewertet werden. Novati hatte dieses Arbeitsprogramm in seiner lectura in Orsanmichele skizziert: «Man hat auch beständig vergessen, den Text der Briefe mit den zwei oder drei Handschriften, die sie überliefern, zu vergleichen« (»Tra l'altro si è costantemente dimenticato di riconfrontare sui due o tre manoscritti che ce ne forniscono il modo, la lezione delle epistole stesse«). Auf dieser Grundlage beurteilt Novati die vulgata, die Torri und Witte erarbeitet hatten und sein Urteil ist vernichtend: In ihrem Versuch, Dantes Text sein ursprüngliches Antlitz zurückzugeben (»al suo naturale colore«), sind die beiden Gelehrten so verfahren wie die inkompetenten Restauratoren der Landkirchen: »Mit jener leichter Hand, mit jener Kenntnis der Schrift der Zeit, mit der häufig verblassten Fresken in Dorfkirchen von stümperhaften Malern ihre ursprüngliche `Farbe` zurückgegeben wird «. ${ }^{44}$ Das Urteil, obwohl hochtrabend formuliert, entbehrte nicht einer soliden Grundlage und was die Briefe Dantes angeht, hätte diese Methode gewiss bedeu-

43 Siehe Luigi Clerici (Hrsg.), »I docenti, i corsi, gli allievi (1861-1915)«, in: Barbarisi/Decleva/ Morgana (Hrsg.), Milano e l'Accademia scientifico-letteraria (wie Anm. 14), S. 1132 und 1083-1185. 44 Novati, Le epistole (wie Anm. 25), S. 26: »con quella leggerezza di mano, quella perizia della grafia del tempo, con cui vediamo spesso essere ricondotti da vaganti impastratori di tele al loro snaturale colore` gli affreschi smunti ed evanescenti di rusticane chiesette«. 
tende Resultate produziert. Wie man weiß, erstellte der neu mit der Edizione nazionale betreute padre Ermenegildo Pistelli in aller Eile einen Text voller Eingriffe. Er war für Novatis Vorschläge völlig unempfänglich; er war ein leichtfertiger »Stümper«.

Übersetzt von Johannes Bartuschat 